\section{Adams-Oliver syndrome with unusual central nervous system findings and an extrahepatic portosystemic shunt}

\author{
Carlos Pérez-García, ${ }^{1}$ \\ Yolanda Ruíz Martín, ${ }^{2}$ \\ Alejandra Aguado del Hoyo, 2 \\ Carlos Marín Rodríguez, 2 \\ Minia Campos Domínguez ${ }^{3}$ \\ 1Department of Radiology, General \\ University Hospital Gregorio Marañón, \\ Madrid; 2Department of Pediatric \\ Radiology, and 3Department of Pediatric \\ Dermatology, Mother and Child \\ Hospital Gregorio Marañón, Madrid, \\ Spain
}

\begin{abstract}
We report a case of a premature neonate girl with scalp and skull defects and brachydactyly of the feet consistent with an Adams-Oliver syndrome (AOS). The patient had central nervous system abnormalities, such as periventricular calcifications, hypoplastic corpus callosum, and bilateral hemispheric corticosubcortical hemorrhagic lesions. A muscular ventricular septal defect and a portosystemic shunt were diagnosed. To our knowledge, this is the first report of congenital supratentorial grey-white matter junction lesions without dural sinus thrombosis in association with AOS. Some of these lesions may be secondary to birth trauma (given the skull defect) whilst others have a watershed location, perhaps as further evidence of vascular disruption and decreased perfusion during critical periods of fetal brain development as the previously proposed pathogenesis of this syndrome.
\end{abstract}

\section{Introduction}

Adams-Oliver syndrome (AOS) is a rare congenital disorder with a wide spectrum of physical anomalies, mainly including aplasia cutis congenita (ACC) and/or bony defects of the underlying skull, associated with various degrees of terminal limb defects. Autosomal dominant inheritance with reduced penetrance and variable expression is most commonly observed, however, sporadic autosomal recessive inheritance has also been reported. Apart from scalp and limb defects, a large number of additional features are reported in AOS. These include cardiac anomalies, renal mal- formations, cleft palate, microphthalmia, skull defects, polythelia, cryptorchidism, cutaneous vascular abnormalities, and lymphatic abnormalities. Central nervous system (CNS) malformations and intellectual disability are present in a few cases.

We report a case of a newborn with autosomal dominant AOS who presented an extrahepatic portosystemic shunt and bilateral cortico-subcortical hemorrhagic contusions/infarcts. To our knowledge these abnormalities have not been reported in association with AOS

\section{Case Report}

We report on a preterm newborn girl (36 weeks and 3 days), small for gestational age $(2.14 \mathrm{~kg})$, the first-born infant of a 23 -yearold woman diagnosed with Adams-Oliver syndrome (AOS) and whose pregnancy was poorly controlled. It was a cephalic-presentation eutocic delivery with normal Apgar score. The newborn was admitted to the neonatology intensive care unit directly from the delivery room with the suspicion of congenital heart disease. Echocardiography was made and demonstrated a large (7-8 $\mathrm{mm})$ muscular ventricular septal defect (VSD) and crossed pulmonary arteries due to an anomalous origin of both pulmonary arteries from the main pulmonary trunk. Physical examination revealed a large parieto-occipital area of hairless atrophic skin surrounded by ulcerated lesions with an underlying bone defect at the patient's vertex. Brachydactyly of both feet was also noted. On the second day of life a transfontanelar ultrasound was performed and depicted multiple bilateral periventricular hyperechogenic nodules. TORCH infections (cytomegalovirus, toxoplasmosis, rubella, herpes simplex and varicella-zoster) were discarded in the serological analysis. On the 22nd day of life a head CT was performed and confirmed the suspicion of periventriucular calcifications (Figure 1). A large parieto-occipital skull defect underlying the aplasia cutis of the vertex was also noted, more apparent on the right side of the skull. In addition, bilateral posterior paramedian parietal lesions in the gray-white matter junction were depicted. These lesions were hypodense (CSF-like), with an appearance of focal loss of tissue as there was enlargement of sulci and gyral thinning. Although these lesions could be secondary to delivery traumatisms (due to the skull defect), since the Adams-Oliver syndrome has been associated with cortical development abnormalities, a cranial MRI was performed on the $23 \mathrm{rd}$ day of life. The
Correspondence: Carlos Pérez-García, Department of Radiology, Hospital General Universitario Gregorio Marañón, Calle del doctor Esquerdo, 46, Madrid, 28007, Spain.

Tel.: +34.649056682 .

E-mail: cperezgarcia.med@gmail.com

Key words: Adams-Oliver syndrome; periventricular calcifications; portosystemic-shunt; pediatric neuroradiology.

Contributions: CPG has done the work of writing the case report and adapt the figures and the text to the specifications of the journal; YRM has been in charge to correct the text and give the approval to the figures; AAdH and CMR have contributed to $\mathrm{CT}$ and MRI images; MCD has contributed to the images of photographs taken in the neonatal ICU.

Conflict of interest: the authors declare no potential conflict of interest.

Received for publication: 5 May 2017

Accepted for publication: 5 June 2017.

This work is licensed under a Creative Commons Attribution NonCommercial 4.0 License (CC BY-NC 4.0).

(C) Copyright C. Pérez-García et al., 2017

Licensee PAGEPress, Italy

Pediatric Reports 2017: 9:7211

doi:10.4081/pr.2017.7211

MRI protocol included sagittal 3D gradientecho T1-weighted images, axial and coronal TSE T2-weighted images, axial FLAIR images, axial T1 phase-sensitive inversionrecovery images, axial susceptibilityweighted images (SWI-BOLD), axial diffusion weighted images (DWI) and sagittal time of flight (TOF) 3D venography. Apart from the findings already described, MRI showed a normal myelination according to age, hypoplastic corpus callosum, dysmorphic lateral ventricles without ventriculomegaly, posterior paramedian parietal hemorrhagic contusions with a cyst-like appearance, distinguishing a fluid-fluid level in the T2-weighted images consistent with a subacute hemorrhagic component. In addition, similar cortico-subcortical lesions were encountered at bilateral parieto-occipital and temporobasal locations (Figure 2). Venography demonstrated permeability of the dural sinuses with a persistent falcine sinus as a variant. At four months of age, an abdomen MRI angiogram was performed and depicted an extrahepatic portosystemic shunt (Abernethy malformation type II) with a dilated vein that originated in the portomesenteric junction and drained into 
the left renal vein (Figure 3). Both the portal venous system and the inferior vena cava had a normal caliber without other abnormalities.

\section{Discussion}

Adams-Oliver syndrome was first described in a male infant in 1945 as an association of a defect in the vertex of the scalp and skull (aplasia cutis congenita) and distal extremity defects. ${ }^{1}$ Original epidemiological data were consistent with a dominant mode of inheritance. Afterwards, both autosomal recessive (multiple reports of

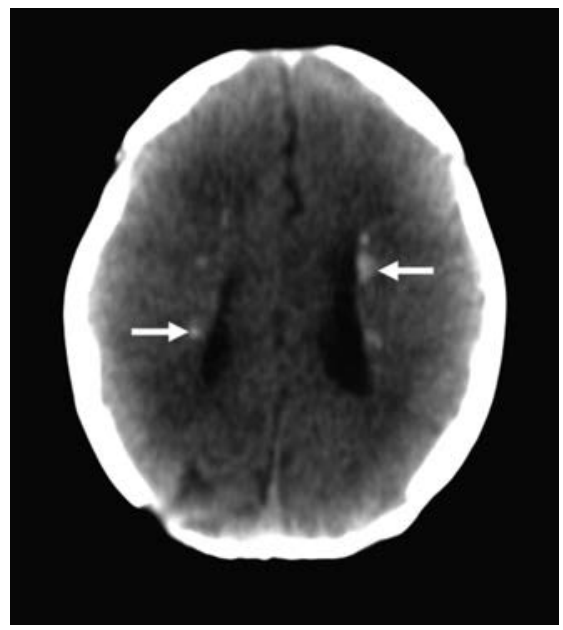

Figure 1. Unenhanced head computed tomography image shows intracranial calcifications in a periventricular distribution (white arrows).

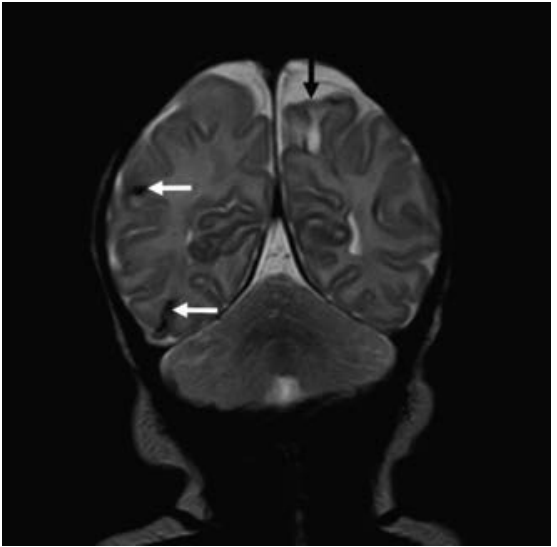

Figure 2. Head magnetic resonance image. Coronal TSE T2-weighted image demonstrates a cyst-like posterior paramedian parietal hemorrhagic contusion (black arrow) and parieto-occipital and temporobasal cortico-subcortical focal areas of low signal intensity (white arrows). recurrence in consanguineous unions) and sporadic occurrences have been reported. Since it was first described, AOS has become recognized as a heterogeneous condition occasionally associated with multiple system anomalies and malformations. In addition, phenotypic variability of this syndrome results in mild to severe defects. ${ }^{2}$

Although the exact pathogenesis of AOS is unknown, the most popular theory is that a vascular disruption or impaired circulation occurs in watershed areas, a genetic defect causing a vasculopathy leading to variable phenotypes. This is further supported by the occurrence of vascular malformations, including congenital cutis marmorata telangiectatica and abnormal pulmonary and portal vasculature in association with AOS. Histological evidence demonstrates an in-utero vascular event leading to interruption of blood supply resulting in phenotypic features. 3,4

Various cardiac malformations have been described, from atrial and ventricular septal defects to aortic coarctation, which makes a cardiac workup mandatory. In our reported case, a large muscular ventricular septal defect was found and in further echocardiogram examinations a dilation of the pulmonary artery trunk $(16 \mathrm{~mm})$ was depicted. Furthermore, our patient was diagnosed of an extrahepatic type 2 portosystemic shunt, also named Abernethy malformation. In this congenital abnormality, the portal vein is intact, but some of the portal flow is diverted usually into the vena cava through a side-to-side extrahepatic communication. It has been proposed that extrahepatic portosystemic shunts originate with the persistence of subcardinohepatic anastomosis with the vitelline veins. ${ }^{5}$ In our case, the shunt consisted in a communica-

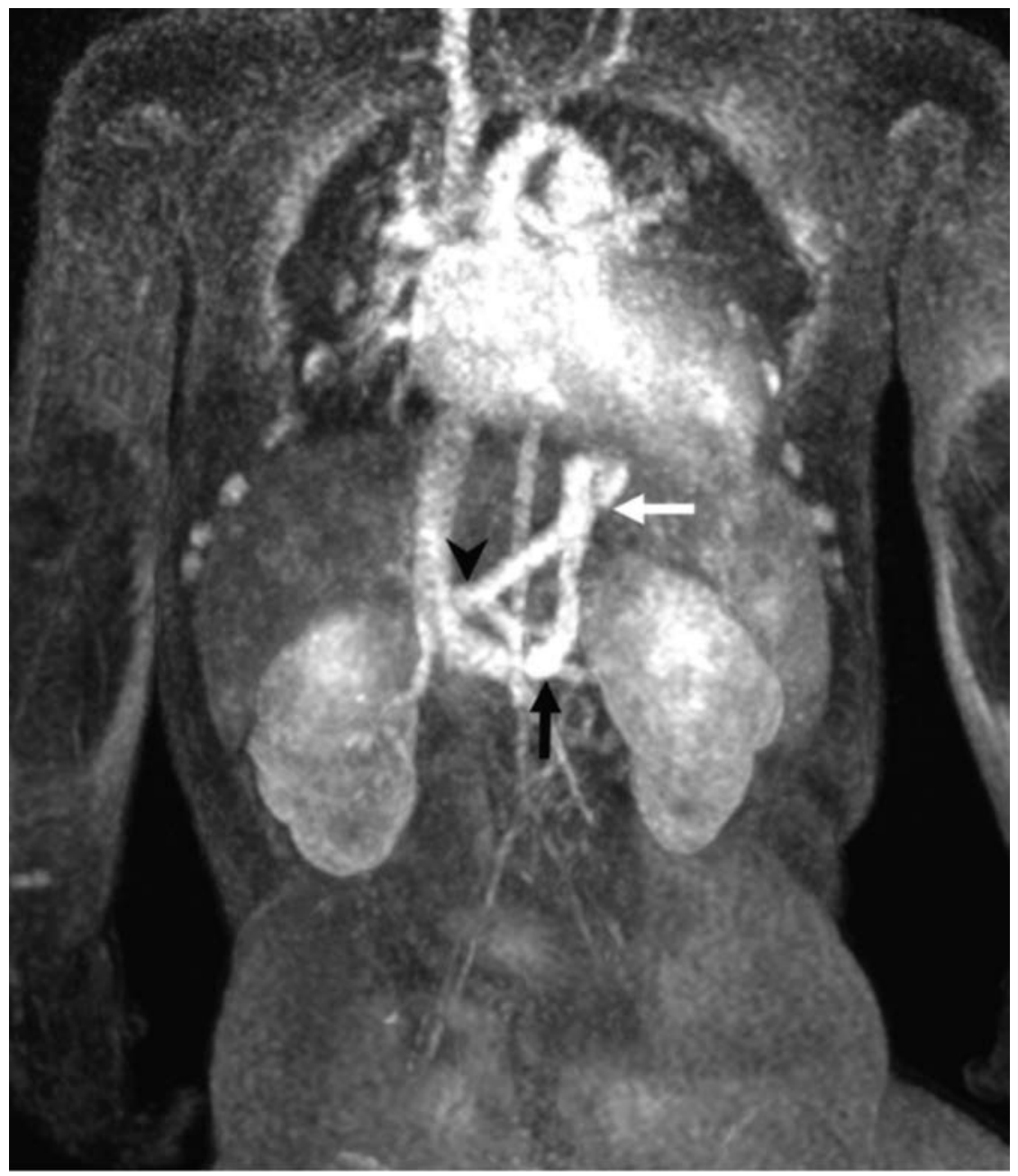

Figure 3. Full body magnetic resonance imaging angiogram image. Coronal maximum intensity projection image in portal venous phase depicts a large vein (white arrow) arising from the left renal vein (black arrow) and draining in the portomesenteric junction (black arrowhead) consistent with an extrahepatic portosystemic shunt. 
tion between the origin of the portal venous trunk (superior mesenteric and splenic veins junction) and the left renal vein through an abnormal dilated vein. To our knowledge this malformation has not been reported yet in association with AOS.

A wide spectrum of intracranial abnormalities has been described in AOS, from milder findings such as idiopathic intracranial calcifications to more severe malformations, including cortical dysplasia, polymicrogyria or acrania. Congenital periventricular calcifications in a pattern similar to those detected in our patient are characteristic of congenital infection by cytomegalovirus or toxoplasma, and they can rarely be caused by rubella, herpesvirus, human immunodeficiency virus (HIV), and other intrauterine infections (TORCH). However, intrauterine infection was not proved in our patient as the serological analysis resulted negative. The periventricular distribution of the calcifications is the most common location described in the literature in association with AOS. ${ }^{6}$ In addition, the cranial MRI showed an abnormally small (hypoplastic), but completely formed, corpus callosum. CT and MRI demonstrated intracranial parenchymal lesions located in the paramedian parietal grey-white matter junction with a cyst-like appearance consistent with subacute hemorrhagic contusions with gliosis, probably secondary to trauma at birth since these lesions were situated underlying the skull defect. Other cortico-subcortical lesions with a similar appearance but smaller in size were encountered bilaterally in parieto-ocipital and temporobasal locations. These lesions were caudal to the skull defect and therefore less likely to be secondary to birth trauma.
Given that the parieto-ocipital and temporobasal grey-white matter junction are watershed areas (border zones between the vascular territories of the medial cerebral artery and posterior cerebral artery) maybe these lesions are secondary to congenital watershed infarctions as a further indication of vascular intrauterine disruption in AOS. Dysplasia of the cerebellar cortex with cysts was described by D'Amico et al. ${ }^{7}$ with a similar appearance to our patient, although no supratentorial lesions were described. Finally, Stittrich et al. ${ }^{8}$ reported multiple cases of AOS including a patient with various infarcts in paramedian parietal and parieto-ocipital locations, however, in that case a superior sagittal sinus thrombosis was detected. In our patient, permeability of the dural venous sinuses was confirmed.

\section{Conclusions}

In a newborn with periventricular calcifications, a pre-natal CMV infection should be ruled out, as well as other TORCH infections. Neonates diagnosed with AdamsOliver syndrome may benefit from imaging tests to rule out CNS, cardiac, renal, and vascular malformations. We believe our case brings in further evidence of vascular disruption and decreased perfusion during critical periods of fetal brain development in AOS.

\section{References}

1. Adams FH, Oliver CP. Hereditary deformities in man: due to arrested development. J Hered 1945;36:3-7.

2. Renfree KJ, Dell PC. Distal limb defects and aplasia cutis: Adams-Oliver syndrome. J Hand Surg Am 2016;41: e207-10.

3. Piazza AJ, Blackston D, Sola A. A case of Adams-Oliver syndrome with associated brain and pulmonary involvement: Further evidence of vascular pathology? Am J Med GenetPart A 2004;130A: 172-5.

4. Al-Sannàa N, Adatia I, Teebi A. Transverse limb defects associated with aorto-pulmonary vascular abnormalities: vascular disruption sequence or atypical presentation of Adams-Oliver syndrome? Am J Med Genet 2000;94: 400-4.

5. Gallego C, Miralles M, Marín C, et al. Congenital hepatic shunts. Radiographics 2004;24:755-72.

6. Balasubramanian M, Collins, AL. Aplasia cutis congenita, terminal limb defects and periventricular leukomalacia in one sibling with minor findings in the other-probable autosomal recessive Adams-Oliver Syndrome. Eur J Med Genet 2009;52:234-8.

7. D'Amico A, Melis D, D'Arco F, et al. Adams Oliver syndrome: Description of a new phenotype with cerebellar abnormalities in a family. Pol J Radiol 2013; 78:83-7.

8. Stittrich AB, Lehman A, Bodian DL et al. Mutations in NOTCH1 cause Adams-Oliver syndrome. Am J Hum Genet 2014;95:275-84. 